This item was submitted to Loughborough's Research Repository by the author.

Items in Figshare are protected by copyright, with all rights reserved, unless otherwise indicated.

\title{
Reduced-order robust tilt control design for high-speed railway vehicles
}

PLEASE CITE THE PUBLISHED VERSION

http://dx.doi.org/10.1080/00423110802037222

PUBLISHER

(C) Taylor \& Francis

VERSION

AM (Accepted Manuscript)

LICENCE

CC BY-NC-ND 4.0

REPOSITORY RECORD

Zolotas, Argyrios C., Jun Wang, and Roger M. Goodall. 2019. "Reduced-order Robust Tilt Control Design for High-speed Railway Vehicles". figshare. https://hdl.handle.net/2134/5505. 
This item was submitted to Loughborough's Institutional Repository (https://dspace.lboro.ac.uk/) by the author and is made available under the following Creative Commons Licence conditions.

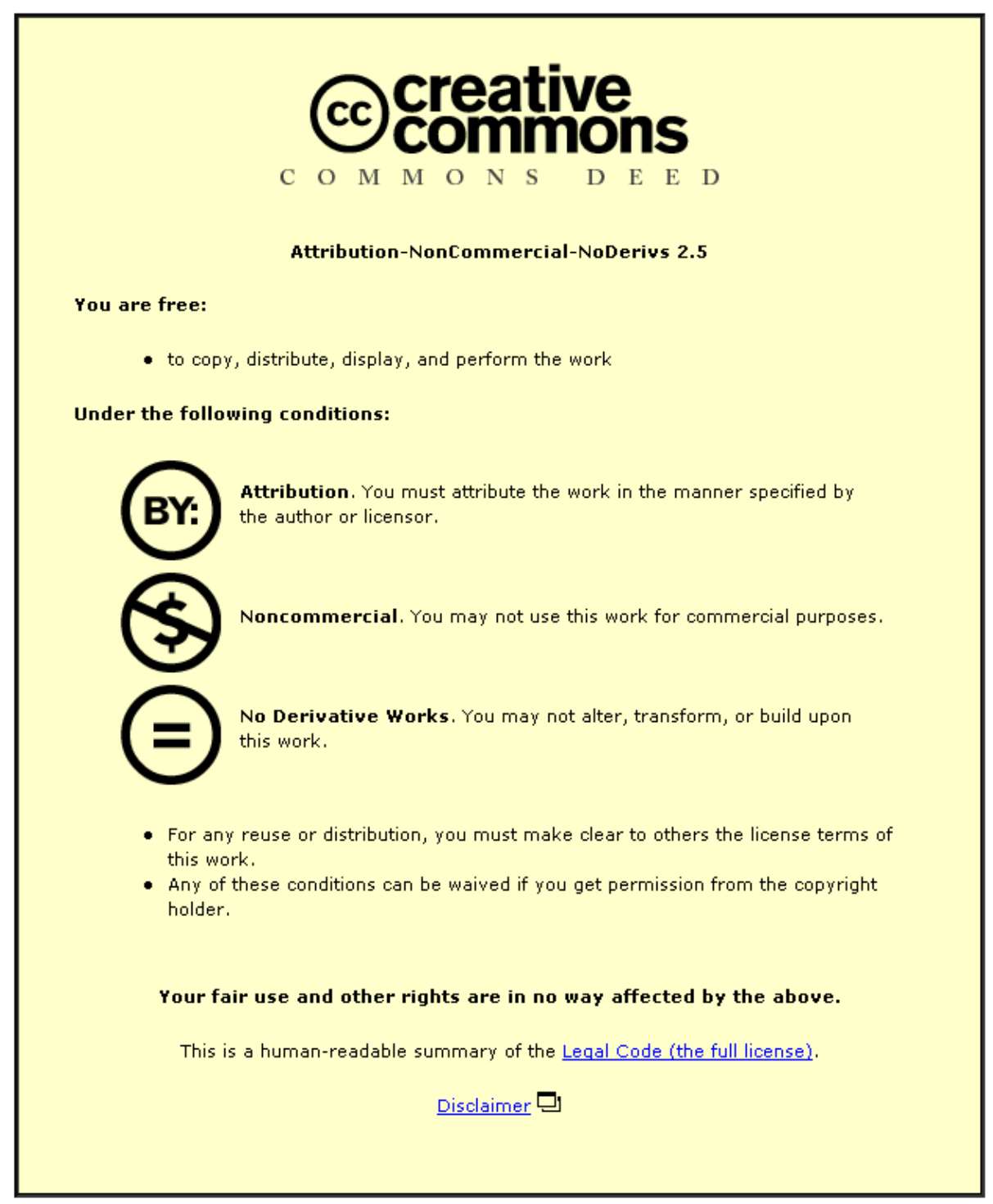

For the full text of this licence, please go to: http://creativecommons.org/licenses/by-nc-nd/2.5/ 


\title{
Reduced Order Robust Tilt Control Design for High Speed Railway Vehicles
}

\author{
Argyrios C. Zolotas* $\dagger$, Jun Wang $\ddagger$, Roger M. Goodall $\dagger$ \\ $\dagger$ Department of Electronic and Electrical Engineering, Loughborough University, UK \\ $\ddagger$ Department of Automation, Tsinghua University, P.R. China
}

\begin{abstract}
This paper presents a study of model reduction techniques, as well as controller reduction via closed-loop considerations in designing robust tilt controllers to improve the curving performance of high speed railway vehicles. The schemes make exclusive use of local practical signal measurements, i.e. sensors mounted on the current passenger coach. The fundamental problem related with straightforward classical nulling-feedback control is presented, while the commercially-used command-driven with precedence scheme is introduced. Simulation results and an appropriately defined tilt control system assessment method are employed for illustrating the efficacy of the reduced-order robust tilt controller.
\end{abstract}

Keywords: Railway vehicles; Tilt control; Robust control; Model reduction; Controller reduction

\section{Introduction}

Active tilting has become well established in modern railway vehicle technology, with most current highspeed train services in Europe now fitted with tilt and an increasing interest for regional express trains [1]. The concept of tilt is rather straightforward, i.e. reduce the passenger lateral acceleration by leaning the vehicle coach inwards on curves, thereby enabling higher vehicle speeds. These were researched in the 1960s and 1970s, developed for production during the 1980s, and increasingly introduced into service operation during the 1990s.

Early tilt control systems were based solely upon local-per vehicle measurements, however it proved difficult at the time to get an appropriate combination of straight track and curve transition performance. Interactions between suspension and controller dynamics (with the sensors being within the control loop) led to control limitations and stability problems (in fact from a control theory point of view the emergence of non-minimum zeros in the system transfer functions placed a limit on system performance). Since then, tilt control systems have evolved in an incremental sense, the end result of which is a control structure not optimised from a system point of view. The industrial norm nowadays is to employ precedence control [1] devised in the early 1980s as part of the Advanced Passenger Train development [2]. In this scheme a bogie-mounted accelerometer from the vehicle in front is used to provide precedence track information (using appropriate inter-vehicle cable/signalling connections), carefully designed so that the filter delay compensates for the preview time corresponding to approximately a vehicle length. Nevertheless achieving a satisfactory local tilt control strategy remains an important research topic because of the system simplifications and more straightforward failure detection.

Observer-based modern control-design methodologies such as LQG, $\mathcal{H}_{\infty}$ are widely used in active suspension design, because they are theoretically well developed, equipped with user-friendly software, and can appropriately deal with active suspension design objectives. However, these methods typically result in controllers of an order comparable to that of the plant (possibly augmented with extra -weighting filters-dynamics). Since a high-order controller is clearly impractical in most situations, e.g. to implement in economical single-chips while satisfying real-time control requirements, some approximation or model reduction techniques are essential.

Few papers have considered the issue of reduced order controller design for active suspensions [4], with

*Corresponding author. Email: a.c.zolotas@lboro.ac.uk Vehicle System Dynamics

Author final version (Vehicle System Dynamics, Vehicle System Dynamics, 2008, 46: 1, 995-1011) 
a recent paper presenting preliminary results on this area specifically for tilt control design [5] and this is the main motivation for carrying out the research presented here. In particular, this paper studies the two reduction approaches, i.e. control design via a reduced-order model and control design via approximation of the high-order controller, for designing simplified robust tilt controllers. The control design method adopted is the LQG with Loop Transfer Recovery (LQG/LTR) to emphasize robustness of the overall LQG controller structure.

The paper is organised as follows: Section 2 presents the tilting train model. Section 3 discusses the concept of model/controller reduction. The control specifications for the tilt controller design as well as the method for assessing its performance are presented in Section 4. The control design procedure with remarks on the individual design approaches is discussed in Section 5, while results are presented and analysed in Section 6. Finally, concluding remarks are made in Section 7.

\section{$2 \quad$ Vehicle modelling}

The modelling of the tilting railway vehicle is based upon a linearised end-view diagram (Figure 1), including both lateral and roll dynamics of both the body and the bogie plus the contribution of the airspring, the dynamics of the actuation system and the bogie lateral kinematics (resulting to a $13^{\text {th }}$ order model). The pair of linear airsprings represents the vertical suspensions, which only contribute to the roll motion of the vehicle (vertical degrees of freedom are ignored). The model also contains the stiffness of an anti-roll bar connected between the body and the bogie frame. Detailed wheelset dynamics were not included for simplicity, however the associated effects are incorporated in the model by using an appropriate 2nd order LP filter (bogie lateral kinematics). The filter was characterised by a $5 \mathbf{H z}$ cut-off frequency and $20 \%$ damping.

Active tilt is provided via a rotational displacement actuator, in series with the roll stiffness ('active anti-roll bar (ARB)' [6]), represented by a position servo in series with the ARB; the parameters were chosen to provide $3.5 \mathrm{~Hz}$ bandwidth and $50 \%$ damping; in addition, the ARB is assumed to provide up to a maximum tilt angle of 10 degrees. The advantages of ARB result from their relative simplicity, i.e. small weight increase, low cost, easily fitted as an optional extra to build or as a retro-fit.

The mathematical models of increasing complexity,via the Newtonian approach, were developed to encapsulate the lateral and roll dynamics of the tilting vehicle system. The equations of motion are given below with all variables and parameter values provided in Appendix A. For the vehicle body (lateral and roll):

$$
\begin{gathered}
m_{\mathrm{v}} \ddot{y}_{\mathrm{v}}=-2 k_{\mathrm{sy}}\left(y_{\mathrm{v}}-h_{1} \theta_{\mathrm{v}}-y_{\mathrm{b}}-h_{2} \theta_{\mathrm{b}}\right)-2 c_{\mathrm{sy}}\left(\dot{y}_{\mathrm{v}}-h_{1} \dot{\theta}_{\mathrm{v}}-\dot{y}_{\mathrm{b}}-h_{2} \dot{\theta}_{\mathrm{b}}\right)-\frac{m_{\mathrm{v}} v^{2}}{R}+m_{\mathrm{v}} g \theta_{\mathrm{o}}-h_{\mathrm{g} 1} m_{\mathrm{v}} \ddot{\theta}_{\mathrm{o}} \\
\begin{array}{r}
i_{\mathrm{vr}} \ddot{\theta}_{\mathrm{v}}=-k_{\mathrm{vr}}\left(\theta_{\mathrm{v}}-\theta_{\mathrm{b}}-\delta_{\mathrm{a}}\right)+ \\
2 h_{1}\left\{k_{\mathrm{sy}}\left(y_{\mathrm{v}}-h_{1} \theta_{\mathrm{v}}-y_{\mathrm{b}}-h_{2} \theta_{\mathrm{b}}\right)+c_{\mathrm{sy}}\left(\dot{y}_{\mathrm{v}}-h_{1} \dot{\theta}_{\mathrm{v}}-\dot{y}_{\mathrm{b}}-h_{2} \dot{\theta}_{\mathrm{b}}\right)\right\} \\
+m_{\mathrm{v}} g\left(y_{\mathrm{v}}-y_{\mathrm{b}}\right)+2 d_{1}\left\{-k_{\mathrm{az}}\left(d_{1} \theta_{\mathrm{v}}-d_{1} \theta_{\mathrm{b}}\right)-k_{\mathrm{sz}}\left(d_{1} \theta_{\mathrm{v}}-d_{1} \theta_{\mathrm{r}}\right)\right\}-i_{\mathrm{vr}} \ddot{\theta}_{\mathrm{o}}
\end{array}
\end{gathered}
$$

For the vehicle bogie (lateral and roll):

$$
\begin{aligned}
m_{\mathrm{b}} \ddot{y}_{\mathrm{b}}=2 k_{\mathrm{sy}}\left(y_{\mathrm{v}}-h_{1} \theta_{\mathrm{v}}-y_{\mathrm{b}}-h_{2} \theta_{\mathrm{b}}\right)+2 c_{\mathrm{sy}}\left(\dot{y}_{\mathrm{v}}-h_{1} \dot{\theta}_{\mathrm{v}}-\dot{y}_{\mathrm{b}}-h_{2} \dot{\theta}_{\mathrm{b}}\right) \\
\quad-2 k_{\mathrm{py}}\left(y_{\mathrm{b}}-h_{3} \theta_{\mathrm{b}}-y_{\mathrm{w}}\right)-2 c_{\mathrm{py}}\left(\dot{y}_{\mathrm{b}}-h_{3} \dot{\theta}_{\mathrm{b}}-\dot{y}_{\mathrm{w}}\right)-\frac{m_{\mathrm{b}} v^{2}}{R}+m_{\mathrm{b}} g \theta_{\mathrm{o}}-h_{\mathrm{g} 2} m_{\mathrm{b}} \ddot{\theta}_{\mathrm{o}}
\end{aligned}
$$




$$
\begin{aligned}
i_{\mathrm{br}} \ddot{\theta}_{\mathrm{b}}=k_{\mathrm{vr}}\left(\theta_{\mathrm{v}}-\theta_{\mathrm{b}}-\delta_{\mathrm{a}}\right)+2 h_{2}\left\{k _ { \mathrm { sy } } \left(y_{\mathrm{v}}-\right.\right. & \left.\left.h_{1} \theta_{\mathrm{v}}-y_{\mathrm{b}}-h_{2} \theta_{\mathrm{b}}\right)+c_{\mathrm{sy}}\left(\dot{y}_{\mathrm{v}}-h_{1} \dot{\theta}_{\mathrm{v}}-\dot{y}_{\mathrm{b}}-h_{2} \dot{\theta}_{\mathrm{b}}\right)\right\} \\
-2 d_{1}\left\{-k_{\mathrm{az}}\left(d_{1} \theta_{\mathrm{v}}-d_{1} \theta_{\mathrm{b}}\right)\right. & \left.-k_{\mathrm{sz}}\left(d_{1} \theta_{\mathrm{v}}-d_{1} \theta_{\mathrm{r}}\right)\right\}+2 d_{2}\left(-d_{2} k_{\mathrm{pz}} \theta_{\mathrm{b}}-d_{2} c_{\mathrm{pz}} \dot{\mathrm{b}}_{\mathrm{b}}\right) \\
& +2 h_{3}\left\{k_{\mathrm{py}}\left(y_{\mathrm{b}}-h_{3} \theta_{\mathrm{b}}-y_{\mathrm{w}}\right)+c_{\mathrm{py}}\left(\dot{y}_{\mathrm{b}}-h_{3} \dot{\theta}_{\mathrm{b}}-\dot{y}_{\mathrm{w}}\right)\right\}-i_{\mathrm{br}} \ddot{\theta}_{\mathrm{o}}
\end{aligned}
$$

for the (additional) airspring state:

$$
\dot{\theta}_{\mathrm{r}}=-\frac{\left(k_{\mathrm{sz}}+k_{\mathrm{rz}}\right)}{c_{\mathrm{rz}}} \theta_{\mathrm{r}}+\frac{k_{\mathrm{sz}}}{c_{\mathrm{rz}}} \theta_{\mathrm{v}}+\frac{k_{\mathrm{rz}}}{c_{\mathrm{rz}}} \theta_{\mathrm{b}}+\dot{\theta}_{\mathrm{b}}
$$

for the ARB actuation system:

$$
\ddot{\delta}_{\mathrm{a}}=-22 \dot{\delta}_{\mathrm{a}}-483.6 \delta_{\mathrm{a}}+483.6 \delta_{\mathrm{a}_{\mathrm{i}}}
$$

and for the bogie kinematics:

$$
\ddot{y}_{\mathrm{w}}=-12.57 \dot{y}_{\mathrm{w}}-987 y_{\mathrm{w}}+987 y_{\mathrm{o}}
$$

These can be represented in the usual state-space form with the state vector $x$ given by

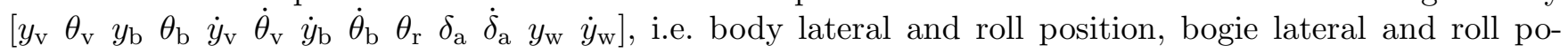
sition, body lateral and roll rate, bogie lateral and roll rate,airspring roll position, applied tilt and tilt rate, bogie kinematics position and rate, respectively (13 states).

It is worth noting that the system is excited by the track disturbance, including both deterministic (low frequency, intended features) and stochastic (higher frequency, track irregularities) elements, while the control input is the ideal tilt command. In fact, for simulation purposes the state vector is augmented with four disturbance states (i.e. track cant position and velocity, track curvature position and straight track position). More details on modelling exercise can be found in [7].

Substantial coupling exists between the lateral and roll motions which result in two sway modes combining both lateral and roll movement. The upper sway mode, its node appears above the body center of gravity, with predominantly roll movement; and the lower sway mode, the node located below the body center of gravity, characterised mainly by lateral motion. The modal analysis of the vehicle is shown in Table 1.

\section{Model and controller reduction concepts}

Model reduction techniques attempt to approximate the dynamic model of the plant by a lower-order system easier to control. In addition, reducing the complexity of the system can offer further advantages, such as elimination of system modes irrelevant to control, simplification of the design process and the accompanying simulations, identification of crucial system characteristics, etc.

Two main approaches exist to the reduced-order controller design problem [3]: (i) direct designs, whereby the low-order controller is designed via an optimization procedure model reduction problem, (ii) and two types of indirect designs. The first type, i.e. model reduction, attempts to approximate the input-output characteristics of the plant by a lower order dynamic system, which automatically results in lower-order controllers when modern-control methods are employed. Of course the approximation should be carried out sensibly, so that the critical modes of the system are not highly affected. For this reason, most modelreduction techniques using this approach are normally accompanied by some form of robust control-design methodology (LQG/LTR, $\mathcal{H}_{\infty} / \mu$ ) to ensure the additional uncertainty introduced by the approximation on system has minimal effects on system stability and performance. The second (indirect) type, i.e. controller reduction, attempts to apply approximation techniques directly to the (high-order) controller. The design concepts can be seen in Figure 2; (note that there are also cases whereby high-order plants and/or reducedorder equivalents can be directly identified from simulation models).

It is worth mentioning that direct low-order controller designs are not as straightforward as the indirect ones, with no commercially available software packages for the design procedure (while a number of software 
packages is available for indirect low-order controller design methods) [3]. The work in this paper has the indirect designs as a central concern.

\subsection{Model reduction}

A number of methods for model reduction are available, i.e. see [3], and the approach followed in this paper is to decompose the system into slow and fast modes, whereby the former is retained and the latter eliminated (effectively keeping a form of the physical interpretation from the original model). The method [8] is briefly described here. Let the original system be given in state-space form as

$$
\dot{x}(t)=A x(t)+B u(t), \quad y(t)=C x(t)+D u(t)
$$

with $A$ stable. Introduce an orthogonal state-space transformation $V$ so that $V^{T} A V$ is in real-Schur form. In this form, the structure of the transformed matrix is essentially upper triangular, with real eigenvalues appearing on the main diagonal, while (simple) complex-conjugate eigenvalues correspond to $2 \times 2$ blocks extending above and below the main diagonal. The eigenvalues (assumed stable) are ordered according to their magnitude, so that the slow modes are located in the upper-diagonal block. Thus, if $m$ represents the number of slow modes that we wish to retain,

$$
\left(\begin{array}{l}
V_{1}^{T} \\
V_{2}^{T}
\end{array}\right) A\left(\begin{array}{ll}
V_{1} & V_{2}
\end{array}\right)=\left(\begin{array}{cc}
A_{s} & A_{12} \\
0 & A_{f}
\end{array}\right)
$$

where $A_{s}$ and $A_{f}$ denote the two blocks containing the slow and fast modes, respectively, and where $V_{1}$ consists of the first $m$ columns of $V$. Note that (simple) complex conjugate eigenvalues are not allowed to be split. Next, let $X$ be the solution of the Sylvester equation

$$
A_{s} X-X A_{f}+A_{12}=0
$$

It is well-known that the solution to this equation exists and is unique, provided that $\lambda_{i}\left(A_{s}\right)-\bar{\lambda}_{j}\left(A_{f}\right) \neq 0$, for all possible $i$ and $j$; note that this is automatically satisfied if the eigenvalues are separated by a positive gap in magnitude as is assumed here. Introducing the additional transformation

$$
\left(\begin{array}{cc}
I & -X \\
0 & I
\end{array}\right)\left(\begin{array}{l}
V_{1}^{T} \\
V_{2}^{T}
\end{array}\right) A\left(\begin{array}{ll}
V_{1} & V_{2}
\end{array}\right)\left(\begin{array}{cc}
I & X \\
0 & I
\end{array}\right)=\left(\begin{array}{cc}
A_{s} & 0 \\
0 & A_{f}
\end{array}\right)
$$

which allows the separation of slow and fast modes via parallel decomposition; and the state vector, in the new coordinate system, is related to the original state vector set $x$ via the transformation

$$
z(t)=\left(\begin{array}{c}
z_{1}(t) \\
z_{2}(t)
\end{array}\right)=\left(\begin{array}{c}
V_{1}^{T}-X V_{2}^{T} \\
V_{2}^{T}
\end{array}\right) x(t)
$$

where $z_{1}(t)$ is the state-vector of the slow part of the realization. This allows us to retain the physical significance of the new state variables $z_{i}$, through their link with the original state-vector $x$. The (loworder) controller can be then designed on the reduced-order model. More details on the actual model approximation of the tilt system are included in the control design section.

\subsection{Controller reduction}

Controller reduction aims to preserve the properties of the closed loop in which the controller is required to operate. This is motivated by the fact that designers desire to have as much closeness as possible in the behavior of the closed-loop of the original plant with the high-order controller and the closed-loop of the original plant with the low-order controller. In particular, controller order reduction may be viewed as 
a frequency-weighted problem, emphasizing the approximation in critical frequency ranges for the closedloop system, e.g. better approximation near the cross-over frequency which is a familiar idea from classical control concepts.

A low-order controller $K_{r}(s)$ is required that will replace the original controller $K(s)$ (designed on the high-order plant) in the closed-loop. This is shown in the top feedback structure of Figure 3 (introduced in an additive sense), while the middle and bottom modified feedback structures in the same figure have the same stability properties, leading to the (most usual) frequency weighted formulation of the controller design. In particular, the reduction problem is to find (a stabilising) low-order controller $K_{r}(s)$ such that the quantity

$$
\left\|\left(K(j \omega)-K_{r}(j \omega)\right) F(j \omega)\right\|_{\infty}
$$

is minimized, with $F(j \omega)=G(j \omega)(I+K(j \omega) G(j \omega))^{-1}$ (the reduction algorithm can be found in [3] and thus we omit its theoretical details $)^{1}$. Effectively, the frequency weight $F(j \omega)$ introduces the importance of the true plant information in the design procedure via the closed loop consideration. The low-order controller $K_{r}(s)$ will then be implemented on the original plant to control.

The two design approaches followed in this paper are summarized in Table 2, whereby (R1) is the loworder design via the reduced order plant (model reduction) and (R2) is the low-order controller design by approximating the high-order controller. Note the fundamental difference between the two design approaches, i.e. plant information is reflected in the (closed-loop based) reduction in design (R2) while this does not apply in design (R1) as approximation occurs early in the design procedure.

\section{Control specifications and assessment method}

The performance of tilt control systems on the curve transitions is critical; most importantly the passenger ride comfort provided by the tilting vehicle should not be (significantly) degraded compared to the nontilting vehicle speeds. The main objectives of any tilt control system are:

(i) to provide an acceptably fast response to changes in track cant and curvature (deterministic track features)

(ii) not to react significantly to track irregularities (stochastic track features)

However, any tilt control system directly controls the secondary suspension roll angle and not the vehicle lateral acceleration. Hence, there is a fundamental trade-off between the vehicle curve transition response and straight track performance. Moreover, for reasons of human perception, designers utilise partial tilt compensation. In such a case the passenger will still experience a small amount of acceleration on steady curve, in order to minimise motion sickness phenomena.

From a control design point of view the objectives of the tilt system can be translated as: increasing the response of the system at low frequencies (deterministic track features), while reducing the high frequency system response (stochastic track features) and maintaining stability.

In particular, the assessment of tilt controllers on curve transition is based upon a combination of the ' $P_{C T}$ factors' and the 'ideal tilting' assessment. The former is based upon a comprehensive experimental/empirical study which provides the percentage of (both standing and seated) passengers who feel uncomfortable during the curve transition. The latter method emphasizes the assessment of the control system performance by determining the deviations from the concept of "ideal tilting", i.e. the tilt action follows the specified tilt compensation in an ideal manner, defined on the basis of the maximum tilt angle and cant deficiency compensation factor. This combination of parameters is optimized via the $P_{C T}$ factors approach to choose a basic operating condition. The procedure follows a minimization approach of dynamic effects relative to tilt angles, roll velocities and lateral accelerations (Figure 4); more details can be found in [9]. The calculation is as follows:

\footnotetext{
${ }^{1}$ Note that there are also alternative frequency weighted formulations with more details also available in [3] and references within.
} 
- $\left|\ddot{y}_{m}-\ddot{y}_{m_{i}}\right|$, the deviation of the actual lateral acceleration $\ddot{y}_{m}$ from the ideal lateral acceleration $\ddot{y}_{m_{i}}$, in the time interval between 1s before the start of the curve transition and 3.6s after the end of the transition.

- $\left|\dot{\theta}_{m}-\dot{\theta}_{m_{i}}\right|$, the deviation of the actual absolute roll velocity $\ddot{\theta}_{m}$ from the ideal absolute roll velocity $\ddot{\theta}_{m_{i}}$, in the time interval between 1s before the start of the curve transition and 3.6s after the end of the transition.

Regarding the straight track case the 'rule-of-thumb' currently followed by designers is to allow a lateral ride quality degradation of the tilting train by no more than a specified margin of between $7.5 \%-10 \%$ compared with the non-tilting vehicle.

\section{Control design}

The control design method adopted is the LQG with Loop Transfer Recovery (LQG/LTR) to emphasize robustness of the overall LQG controller structure, as well as being an attractive (first) design approach in the framework of reduced-order control procedures.

\subsection{LQG control synthesis with loop transfer recovery (LTR)}

Linear Quadratic Gaussian control is well documented (see $[10,11]$ ) and defines the following state-space plant model

$$
\begin{aligned}
\dot{x} & =A x+B u+\Gamma w \\
y & =C x+v
\end{aligned}
$$

where $w, v$ are (ideally) white uncorrelated process and measurement noises that excite the system, and are characterized by covariance matrices $W, V$ respectively. The separation principle (Figure $5(\mathrm{~b})$ ) can be applied to first find the optimal control $u=-K_{c} x$ which minimizes (15)

$$
J=\lim _{T \rightarrow \infty} \frac{1}{T} E\left\{\int_{0}^{T}\left[x^{T} Q x+u^{T} R u\right] d \tau\right\}
$$

where $K_{c}=R^{-1} B^{T} X$ and $X$ is the positive semi-definite solution of the following Algebraic Riccati Equation (ARE)

$$
[X-I]\left[\begin{array}{cc}
A-B R^{-1} B^{T} \\
-Q & -A^{T}
\end{array}\right]\left[\begin{array}{l}
I \\
X
\end{array}\right]=0
$$

Next find the optimal state estimate $\hat{x}$ of $x$ where

$$
\hat{x}=A \hat{x}+B u+K_{f}(y-C \hat{x})
$$

to minimise $E\left\{[x-\hat{x}]^{T}[x-\hat{x}]\right\}$. The optimal Kalman gain is given by $K_{f}=Y C^{T} V^{-1}$ and $Y$ is the positive semi-definite solution of the following ARE

$$
[Y-I]\left[\begin{array}{cc}
A^{T} & -C^{T} V^{-1} C \\
-\Gamma W \Gamma^{T} & -A
\end{array}\right]\left[\begin{array}{c}
I \\
Y
\end{array}\right]=0
$$


Weighting matrices $Q$ (positive semidefinite), $R$ (positive definite.) for control, and $W$ (positive semidefinite), $V$ (positive definite) for estimation, can be tuned to provide the desired result. Note that it is also possible to follow the dual procedure, i.e. solve for the state estimate sub-problem and next for the optimal gain sub-problem.

The synthesized LQG controller transfer function realization (Figure 5(a)) is given by

$$
K_{l q g}(s)=-K_{c}\left(s I-\left(A-B K_{c}-K_{f} C\right)\right)^{-1} K_{f}
$$

Unfortunately the LQG compensators do not exhibit the good robustness properties of both the LQR and Kalman filter [10]. However, there is a way of either designing the Kalman filter such that the LQR robustness properties are 'recovered' at the plant input; or designing the LQR such that the Kalman filter robustness properties are recovered at the plant output. The Loop Transfer Recovery procedure is extensively discussed in [10] and references within.

The main points of the procedure [10] are summarised below (with respect to Figure 5)

(i) Recovery at plant input. Design the Kalman filter gain $K_{f}$ such that the loop TF $K(s) G(s)$ (point 1) approaches $K_{c}(s I-A)^{-1} B$ (point 3 ). The plant must have at least as many outputs as inputs. In order for LTR to be applied, fictitious inputs must be included to make the system square and minimum phase. Recovery can be followed at plant input but not plant output. This is the approach undertaken in this paper.

(ii) Recovery at plant output. Design the LQR gain $K_{c}$ such that the loop TF $G(s) K(s)$ (point 2) approaches $C(s I-A)^{-1} K_{f}$ (point 4$)$. In this case, artificial outputs must be included to make the system square and minimum phase. However, recovery can be applied only at plant output and not plant input.

In both cases the plant is assumed to be minimum phase for full recovery, however in the case of non-minimum phase systems, the same procedure can be used but only to partially recover the required robustness properties for a specific range of frequencies (only partial recovery is achieved even in the case of minimum phase systems in a practical implementation). More details on LTR for non-minimum phase systems can be found in [10]. Note that LTR is a virtual design procedure (using fictitious noise inputs), thus extra care should be taken when recovering the required robustness properties. Also, care should be taken for the required level of robustness to be achieved, due to the fact that full recovery would undoubtedly deteriorate the nominal performance of the true noise problem.

\subsection{Remarks on the tilt control design}

The control design is based on the SISO tilt model (actuator angle $u=\delta_{a_{i}}$ to effective cant deficiency (combination of lateral acceleration and tilt angle) for $60 \%$ tilt compensation on steady curve $\theta_{\text {ecd }}$ ), with all disturbance signals set to zero. It is worth mentioning that, the simulation model (including track disturbance states) is 17th order while the design model for the process in the paper (without disturbance states) is 13th order. Moreover, the LQG/LTR design on the SISO model can be seen as a simple extension of the conventional (classical) nulling tilt problem in the optimal control framework.

The view adopted here is to synthesize the tilt controller via LQG theory with the weighting matrices $Q, R$ for control and $W, V$ for estimation purely considered as tuning parameters until an appropriate design is obtained (recovery at plant input as discussed above) [10]. More importantly,

- First the model is augmented using an extra state (integral of error of effective cant deficiency) for integral action (thus providing zero sensitivity at zero frequencies and zero steady-state errors), i.e.

$$
\begin{aligned}
{\left[\begin{array}{c}
\dot{x} \\
\dot{x}_{i}
\end{array}\right] } & =\left[\begin{array}{cr}
A & 0 \\
-C_{i} & 0
\end{array}\right]\left[\begin{array}{c}
x \\
x_{i}
\end{array}\right]+\left[\begin{array}{c}
B \\
0
\end{array}\right] u+\left[\begin{array}{l}
0 \\
1
\end{array}\right] r \\
y & =\left[\begin{array}{ll}
C_{i} & 0
\end{array}\right]\left[\begin{array}{c}
x \\
x_{i}
\end{array}\right],
\end{aligned}
$$


where $C_{i}$ is the selector matrix for the effective cant deficiency output, and $r$ will be a zero reference in the tilt control case (i.e. regulation).

- An LQR control law is designed using $Q=C_{i}^{T} q_{0} C_{i}+q I$ state weight (selecting the integral state) for speed of response, while $q$ places additional emphasis on the remaining states effectively dampening the response, while the control weight $R=1$ is fixed. Note this is followed for both reduced order controller cases.

- $q 0$ and $q$ have been tuned to 10 and 0.5 respectively to provide fast return ratios in both Reduced Model and Reduced Controller design cases.

- the Kalman filter is designed by assuming virtual process noise entered via the control input (via the $B$ matrix) to recover the robust LQR properties at the input of the plant. The process noise covariance was chosen as $W=W_{0}+w I$ with $W_{0}=1$ and $w$ increasing up to recover sufficient return ratio properties $(\mathrm{w}=50)$, while the sensor noise covariance $V=0.1$ was fixed.

- the SISO design model has non-minimum phase zeros which limit the control performance (with the achievable bandwidth of the system being much less than half of the RHP zero frequency [11]). Thus, partial recovery can be achieved due to the non-minimum phase characteristics.

\subsection{Design $R 1$ - reduced model}

In this approach (see Table 2) a reduced order model $G_{r}$ is found from the original plant $G$ such that $\left\|G-G_{r}\right\|_{\infty}$ is small. For the tilt model a number of model approximations (2nd, 4th, 6th,7th order) are shown in Figure 6(a) compared to the 13th order design model, while the relevant additive errors are shown in Figure 6(b). These are the basis of deciding on the most appropriate choice. Large errors can be seen for the 2nd and 4th order model approximation, with the 6th order approximation being an appropriate choice as it has virtually no difference with the 7 th order equivalent and is also very close to the original model up to 100rad/s.

A 7th order controller is then designed following the Linear Quadratic Gaussian with Loop Transfer Recovery (LQG/LTR) procedure described above, due to the augmentation of the system with the integral state (six states from the chosen reduced model plus one from the additional integral state). The achieved return ratio can be seen in Figure 7 (the non-minimum phase is a strict constraint and no more recovery applies).

\subsection{Design R2 - controller reduction}

As mentioned in Table 2, the high-order controller is designed upon the 13th order design model via the LQG/LTR method mentioned above (in fact the 13th order model includes 2 uncontrollable/unobsvervable states and an 11th order equivalent is directly obtained via minimal realization). The high-order controller size is thus 12 states (due to the augmentation of the minimal realized model with the extra integral state).

Frequency weighted controller reduction was applied to the 12th order controller to retain closed loop performance/stability, with the lowest possible controller order achieved being 5 th. Again partial recovery was achieved due to the non-minimum phase characteristics of the plant but it was still appropriate for control performance. The achieved return ratios can be seen in Figure 8 both for the 12th order and 5th order controllers.

\section{Results and analysis}

Figure 9 illustrates the gain plots of the closed loop transfer function $G(s) K(s)(I+G(s) K(s))^{-1}$ and $G(s) K_{r}(s)\left(I+G(s) K_{r}(s)\right)^{-1}(G(s)$ being the 13th order design model) with the 12th $K(s)$, and 7th and 5 th order $K_{r}(s)$ controllers. All controllers have very similar performance up to $60 \mathrm{rad} / \mathrm{s}$, while the 5 th order controller has lower gains at high frequencies (this suggests better robustness properties to unstructured uncertainty).

In addition to the frequency domain results (including the ones from the previous section), the de- 
signed controllers were validated via simulation (on the 17 th order simulation model with realistic track disturbance signals) using

(i) a curved (design) track of: $1000 \mathrm{~m}$ curve radius, $155 \mathrm{~mm}$ (6deg) track elevation and $145 \mathrm{~m}$ transition at each end of the curve;

(ii) a non-tilting speed of $45 \mathrm{~m} / \mathrm{s}$ and a tilting speed of $58 \mathrm{~m} / \mathrm{s}$ (30\% increase);

(iii) a stochastic (lateral) track irregularities profile chosen as a usual medium quality track with $0.33 \times$ $10^{-8} \mathrm{~m}$ track roughness.

The time domain results for the passenger acceleration and body roll angle profile with all designed controllers validated can be seen in Figure 10, while the detailed performance assessment of the controllers is shown in Table 3. All controllers have similar performance (as expected from the frequency domain results), with the 5th order controller an appropriate choice for implementation purposes due to being the simplest solution. Note that the same weights were used in both design cases, thus some refinement will slightly improve the 12 th and 5 th order controller performance from controller reduction (the aim here was to emphasise the design procedures rather than strictly the choice of weights). Moreover,

- the R1 design based controller performance deteriorates if reduced below 7th order in an open loop sense (especially the stochastic criterion of $7.5 \%$ degradation is violated);

- a further 'closed-loop' reduction process can be applied to the 7th order R1 design based controller to get a 5th order equivalent, but still cannot achieve the performance of the R2 design based 5th order controller (esp. the stochastic performance);

- the R2 design based 5th order controller stochastic performance deteriorates if reduced to 4th order, and stability issues arise if reduced further;

- the R2 design approach is a better choice as it is based on closed loop properties, i.e. the true plant is relevant in the design process in this case, while this does not apply in case R1.

\section{Conclusions}

The paper discussed on the design of reduced-order robust controllers for tilting railway vehicles. The design is based on practical measurements using local vehicle information, i.e. with no 'a priori' information on track profile. Two design approaches were presented, the first on reduced-order controller via model approximation which resulted to a 7th order controller and the second on controller reduction of the highorder controller which resulted to a 5th order controller. All controller structures provided appropriate tilt performance (although subject to non-minimum phase limitation), with the 5th order being the (simplest) choice to implement on the true plant. Shaping the principal gains of the return ratio of the system with the automatic LQG/LTR procedure, avoids manually designing networks of classical compensators. A careful selection and integrated application of different reduction methods can achieve appropriate system performance with low-order controllers, while controller reduction on high-order controllers to achieve appropriate closed loop properties is preferable. The presented approach can be also utilised for H-infinity control methods; current work is based on this as well as robustness assessment via uncertainty representations. The paper should be of considerable interest to control engineers who have to provide practical solutions but may be put off by the potential complexity of normal model-based control techniques. 


\section{Appendix A: Parameter values and notation}

$y_{\mathrm{v}}, y_{\mathrm{b}}, y_{\mathrm{w}}, y_{\mathrm{o}}$ Lateral displacem. of body, bogie, wheelset, track

$\theta_{\mathrm{v}}, \theta_{\mathrm{b}}, \theta_{\mathrm{r}}$

$\delta_{\mathrm{a}}, \delta_{\mathrm{a}_{\mathrm{i}}}$

Roll displacement of body, bogie, airspring reservoir

$\theta_{\mathrm{O}}, R$

ARB applied tilt (actual) and tilt command (ideal)

$v$

Track cant, curve radius

$m_{\mathrm{v}}$

Vehicle forward speed

$i_{\mathrm{vr}}$

Half body mass, 19,000(kg)

$m_{\mathrm{b}}$

Half body roll inertia, 25,000 $\left(\mathrm{kgm}^{2}\right)$

$i_{\mathrm{br}}$

Bogie mass, 2,500 $(\mathrm{kg})$

$g \quad$ gravitational acceleration, $9.81\left(\mathrm{~ms}^{-2}\right)$

(the values below correspond to per bogie side)

$k_{\mathrm{az}}$

$k_{\mathrm{SZ}}$

$k_{\mathrm{rZ}}$

$c_{\mathrm{rz}}$

$k_{\mathrm{sy}}$

$c_{\mathrm{sy}}$

$k_{\mathrm{vr}}$

$k_{\mathrm{pz}}$

$c_{\mathrm{pz}}$

$k_{\mathrm{py}}$

$c_{\text {py }}$

$d_{1}$

$d_{2}$

$h_{1}$

$h_{2}$

$h_{3}$

$h_{\mathrm{g} 2}$

$h_{\mathrm{g} 1}$
Airspring area stiffness, 210,000 $\left(\frac{\mathrm{N}}{\mathrm{m}}\right)$

Airspring series stiffness, 620,000 $\left(\frac{N}{m}\right)$

Airspring reservoir stiffness, 244,000 $\left(\frac{N}{m}\right)$

Airspring reservoir damping, 33,000( $\left.\frac{\mathrm{Ns}}{\mathrm{m}}\right)$

Secondary lateral stiffness, 260,000 $\left(\frac{N}{m}\right)$

Secondary lateral damping, 33,000( $\left.\frac{\mathrm{Ns}}{\mathrm{m}}\right)$

Anti-roll bar stiffness/bogie, 2,000,000 $\left(\frac{\mathrm{Nm}}{\mathrm{rad}}\right)$

Primary vertical stiffness, 2,000,000 $\left(\frac{N}{m}\right)$

Primary vertical damping, 20,000 $\left(\frac{N s}{m}\right)$

Primary lateral stiffness, 35,000,000 $\left(\frac{N}{m}\right)$

Primary lateral damping, 16,000( $\left.\frac{\mathrm{Ns}}{\mathrm{m}}\right)$

Airspring semi-spacing, 0.90(m)

Primary vertical suspension semi-spacing, 1.00(m)

Secondary lateral susp. height(body cog), 0.9(m)

Secondary lateral susp. height(bogie cog), 0.25(m)

Primary lateral susp. height(bogie cog), -0.09(m)

Bogie cog height(rail level), 0.37(m)

Body cog height(rail level), 1.52(m)

\section{References}

[1] Goodall, R.M., 1999, Tilting Trains and Beyond - the Future for Active Railway Suspensions: Part 1 Improving passenger comfort, IEE Journal of Computing and Control Engineering, August, 153-160.

[2] Boocock, D., and King, B.L., 1982, Development of the Prototype Advanced Passenger Train, Proceedings of the Institution of Mechanical Engineers (IMECHE), 196, 35-46.

[3] Obinata, G., Anderson, B.D.O., 2001, Model Reduction for Control System Design, Springer Verlag, New York.

[4] Wang, J., Zolotas, A.C., and Wilson, D.A., 2007, Active Suspensions: A Reduced-Order $\mathcal{H}_{\infty}$ Control Design Study, In Proceedings of the 15th Mediterranean Conference on Control and Automation, Athens, Greece.

[5] Zolotas, A.C., Halikias, G.D., Goodall, R.M., and Wang, J., 2006, Model Reduction Studies in LQG Optimal Control Design for High-Speed Tilting Railway Carriages, In Proceedings of the American Control Conference (Silver Anniversary), Minneapolis, MN, USA.

[6] Pearson, J.T., Goodall, R.M., and Pratt, I., 1998, Control System Studies of an Active Anti-Roll Bar Tilt System for Railway Vehicles, Proceedings of the Institutions of Mechanical Engineers Part F, 212:F1, 43-60.

[7] Zolotas, A.C., and Goodall, R.M., 2000, Advanced Control Strategies For Tilting Railway Vehicles, In Proceedings of the UKACC Control 2000 Conference, Cambridge, UK.

[8] Safonov, M.G., Jonckheere, E.A., Verma, M., and Limebeer, D.J.N., 1987, Synthesis of Positive Real Multivariable Feedback Systems, Int. J. Control, 45:3, 817-842.

[9] Goodall, R.M., Zolotas, A.C., and Evans, J., 2000, Assessment of the Performance of Tilt System Controllers, RAILTEX 2000, NEC Birmingham, UK, 21-23 November.

[10] Maciejowski, J.M., 1989, Multivariable Feedback Design, Addison-Wesley.

[11] Skogestad, S., and Postlethwaite, I., 2000, Multivariable Feedback Control: Analysis and Desgn, Wiley. 
Table 1. $13^{\text {th }}$ order ARB vehicle modal analysis(*)

\begin{tabular}{lrr}
\hline Mode & Damping (\%) & Frequency $(\mathbf{H z})$ \\
\hline Body lower sway & 16.5 & 0.67 \\
Body upper sway & 27.2 & 1.50 \\
Bogie lateral & 12.4 & 26.80 \\
Bogie roll & 20.8 & 11.10 \\
Bogie kinematics & 20.0 & 5.00 \\
Actuation system & 50.0 & 3.50 \\
Airspring mode & 100.0 & 3.70 \\
\hline
\end{tabular}

(*)without disturbance states and before minimal realisation.

\section{Tables with captions}


Table 2. Indirect reduced-order controller procedures for the tilt control exercise

R1: Reduced controller design via low-order plant

"Reduced model"

- approximate original plant $G(s)$ by reduced-order $G_{r}(s)$ such that

$$
\left\|G(j \omega)-G_{r}(j \omega)\right\|_{\infty}
$$

is small

- design the (low-order by default) controller $K_{r}(s)$ on $G_{r}(s)$

- implement $K_{r}(s)$ on $G(s)$
R2: Controller reduction on high-order controller "Reduced Controller"

- design $K(s)$ on original plant $G(s)$

- approximate $K(s)$ by a lower-order $K_{r}(s)$ such that

$$
\left\|\left(K(j \omega)-K_{r}(j \omega)\right) F(j \omega)\right\|_{\infty}
$$

is small, where

$F(j \omega)=G(j \omega)(I+K(j \omega) G(j \omega))^{-1}$

- implement $K_{r}(s)$ on $G(s)$ 
Table 3. Tilting performance assessment (controllers)

\begin{tabular}{|c|c|c|c|}
\hline & $/ 12$ th order $(*)$ & $/ 7$ th order $(\mathrm{R} 1)$ & $/ 5$ th order $(\mathrm{R2})$ \\
\hline \multicolumn{4}{|l|}{ Deterministic- } \\
\hline rms of passenger accel. error (\%g) & 3.55 & 3.28 & 3.42 \\
\hline max passenger accel. value $(\% \mathrm{~g})$ & 15.30 & 14.18 & 14.91 \\
\hline $\max$ jerk level for $\mathrm{P}$ factor $(\% \mathrm{~g} / \mathrm{s})$ & 8.62 & 8.35 & 8.69 \\
\hline$\times 10^{-2} \mathrm{rms}$ of roll rate error $(\mathrm{rad} / \mathrm{s})$ & 3.40 & 3.30 & 3.30 \\
\hline$\times 10^{-2}$ max roll rate value $(\mathrm{rad} / \mathrm{s})$ & 11.30 & 11.70 & 11.40 \\
\hline P-factor standing $\%$ of passengers & 62.43 & 59.91 & 61.76 \\
\hline P-factor seated $\%$ of passengers & 18.26 & 17.16 & 18.03 \\
\hline \multicolumn{4}{|l|}{ Stochastic- } \\
\hline ride quality non-tilting (\%g) & 3.89 & 3.89 & 3.89 \\
\hline ride quality tilting $(\% \mathrm{~g})$ & 4.00 & 4.04 & 4.03 \\
\hline degradation $(\%)$ & 2.92 & 3.86 & 3.55 \\
\hline
\end{tabular}

$(*)$ designed on minimal realisation plant but without further reductions. 


\section{Figure captions}

Figure 1. Tilting vehicle end-view diagram with actuation system;

Figure 2. Reduced order controller design paths;

Figure 3. Reduced-order controller feedback formulation for stability criteria;

Figure 4. "Ideal Tilting"- Calculation of deviation of actual from ideal responses for acceleration and roll velocity;

Figure 5. LQG control design: (a) LQG controller (b) separation principle ;

Figure 6. Model approximation: (a) magnitude of (SISO) approximation models (b) additive reduction errors;

Figure 7. Loop transfer recovery at plant input for design R1;

Figure 8. Loop transfer recovery at plant input for design R2;

Figure 9. Closed loop gain comparisons of the different controller designs;

Figure 10. Time-domain results for design (curved) track (a) passenger acceleration (b) body roll angle (tilt). 


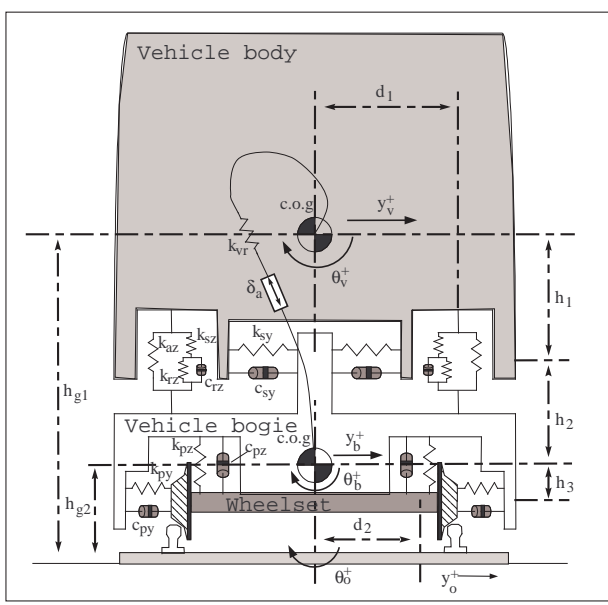

a) End-view of vehicle structure with body, bogie and airspring present

Figure 1

(b) ARB dynamics (position servo)

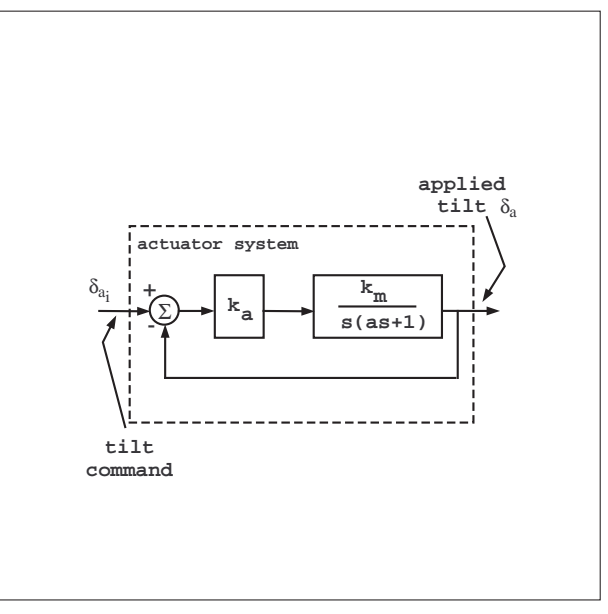

(b) ARB dynamics (position servo) 


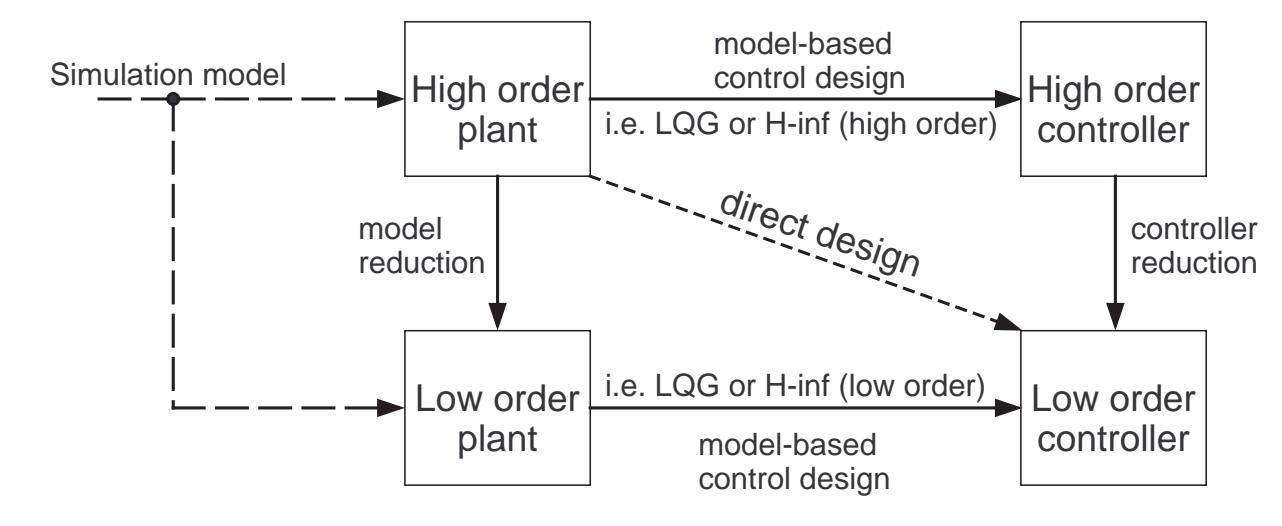

Figure 2

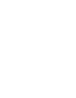



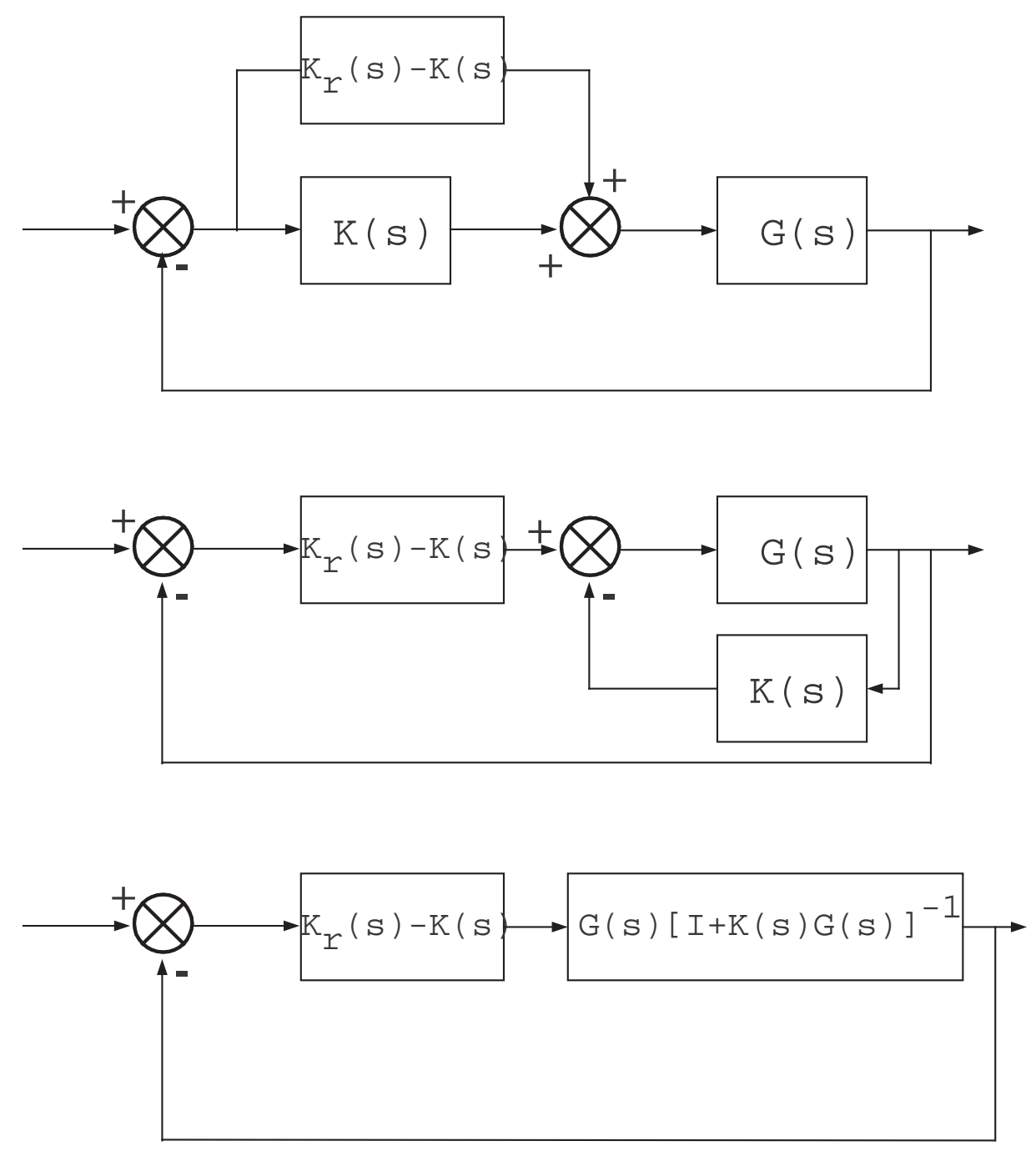

Figure 3 


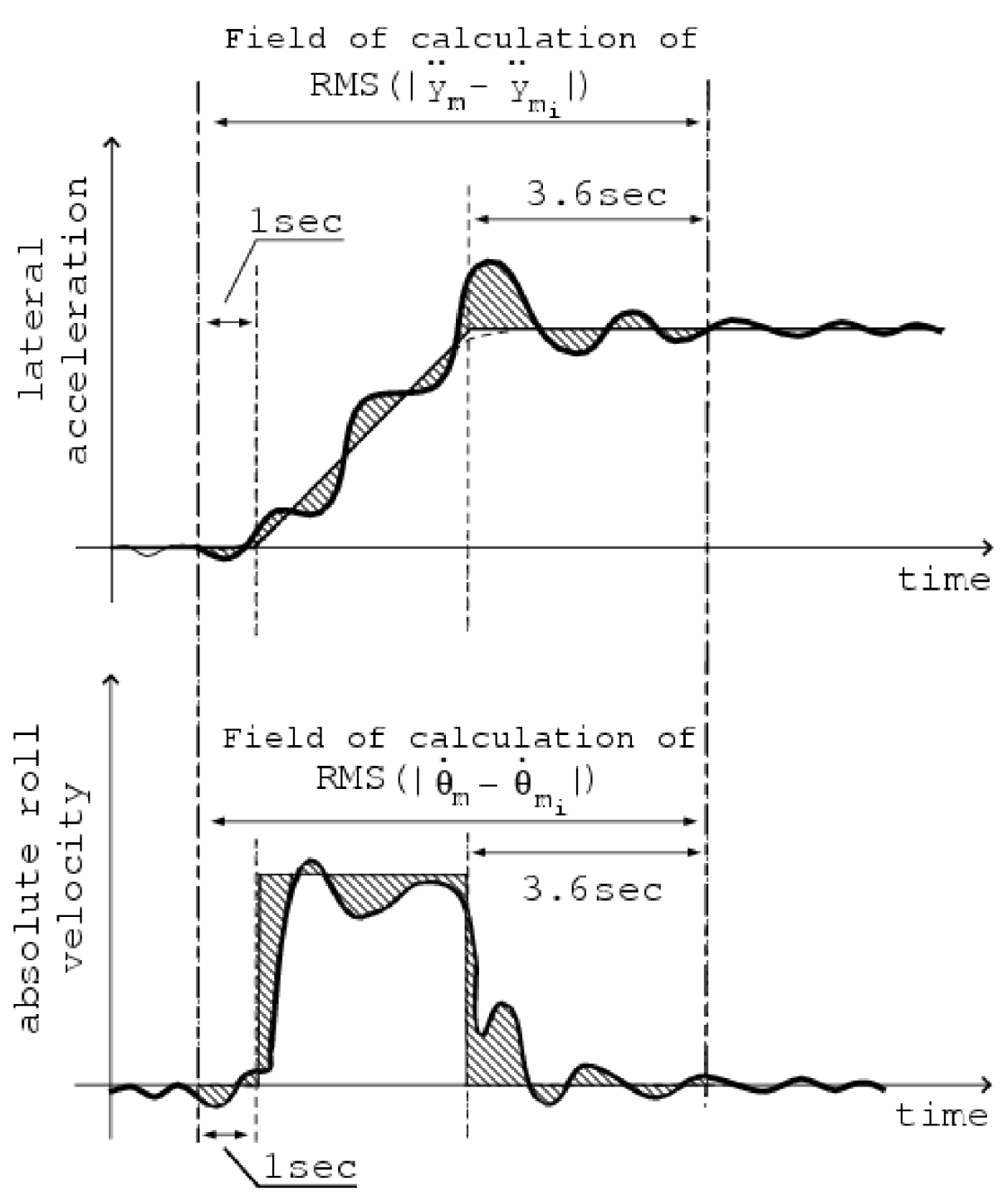

Figure 4 


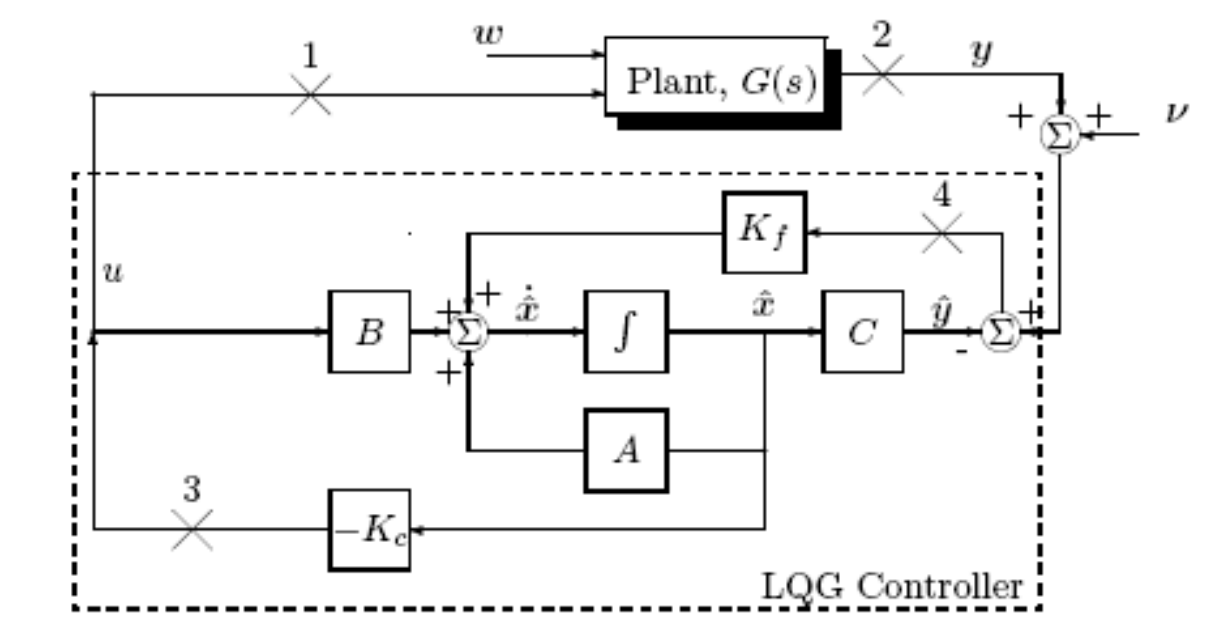

Figure 5 a 


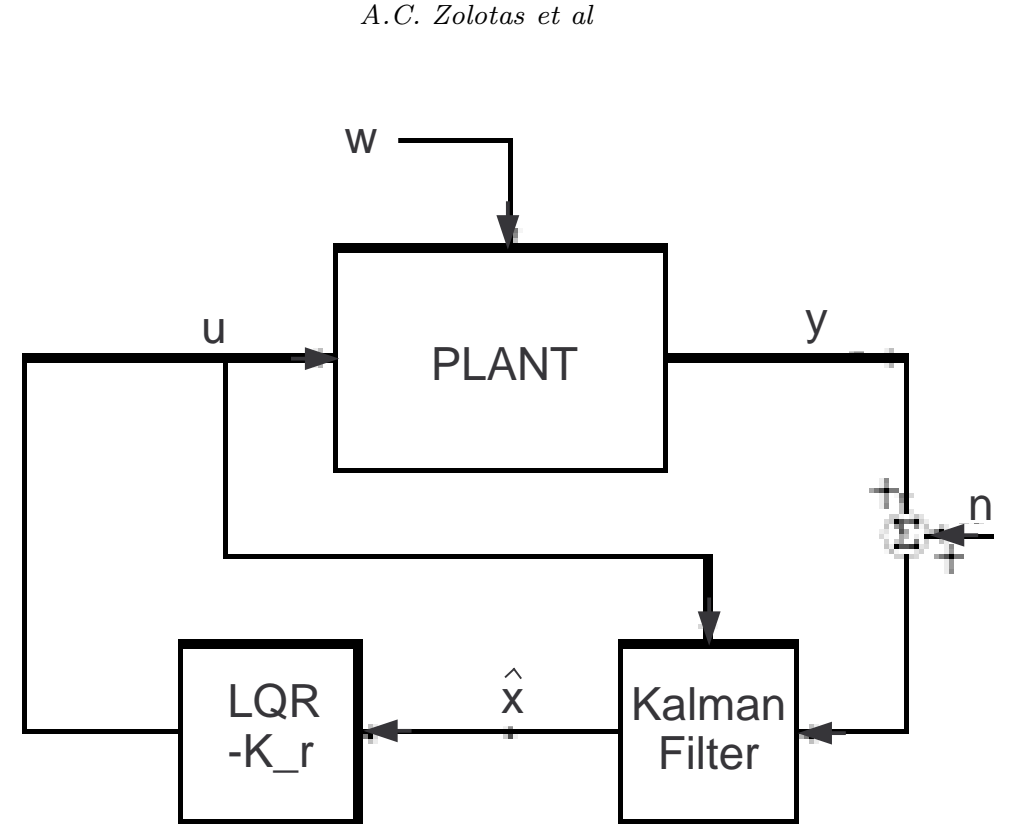

Figure 5b

\author{
Figure 5 b
}


Singular Values

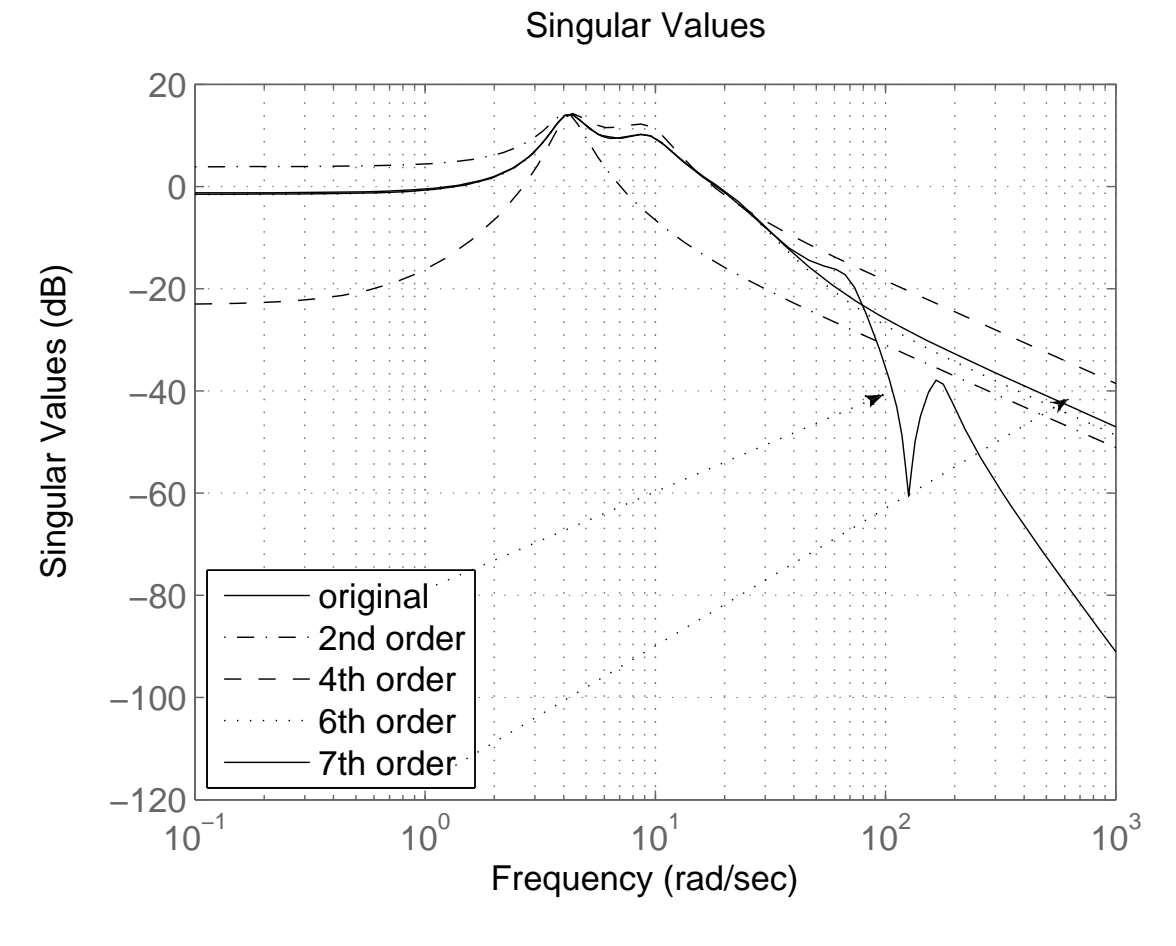

Figure 6a

Frequency (rad/sec)

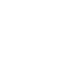

(1)

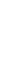


Additive approximation errors $G_{-} G_{r}$

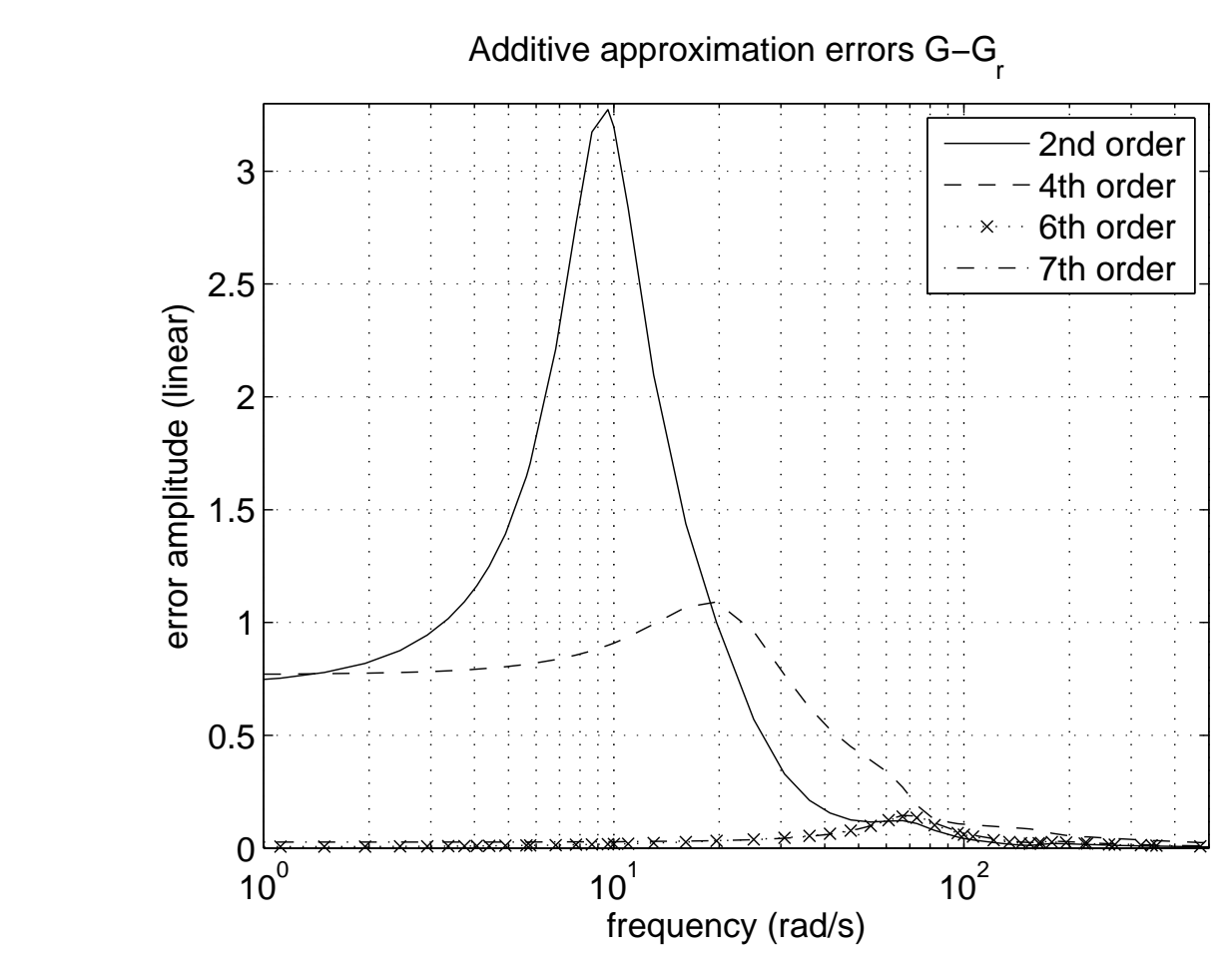

Figure $6 \mathrm{~b}$

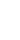

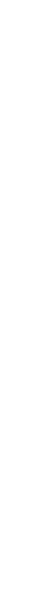

(a) 


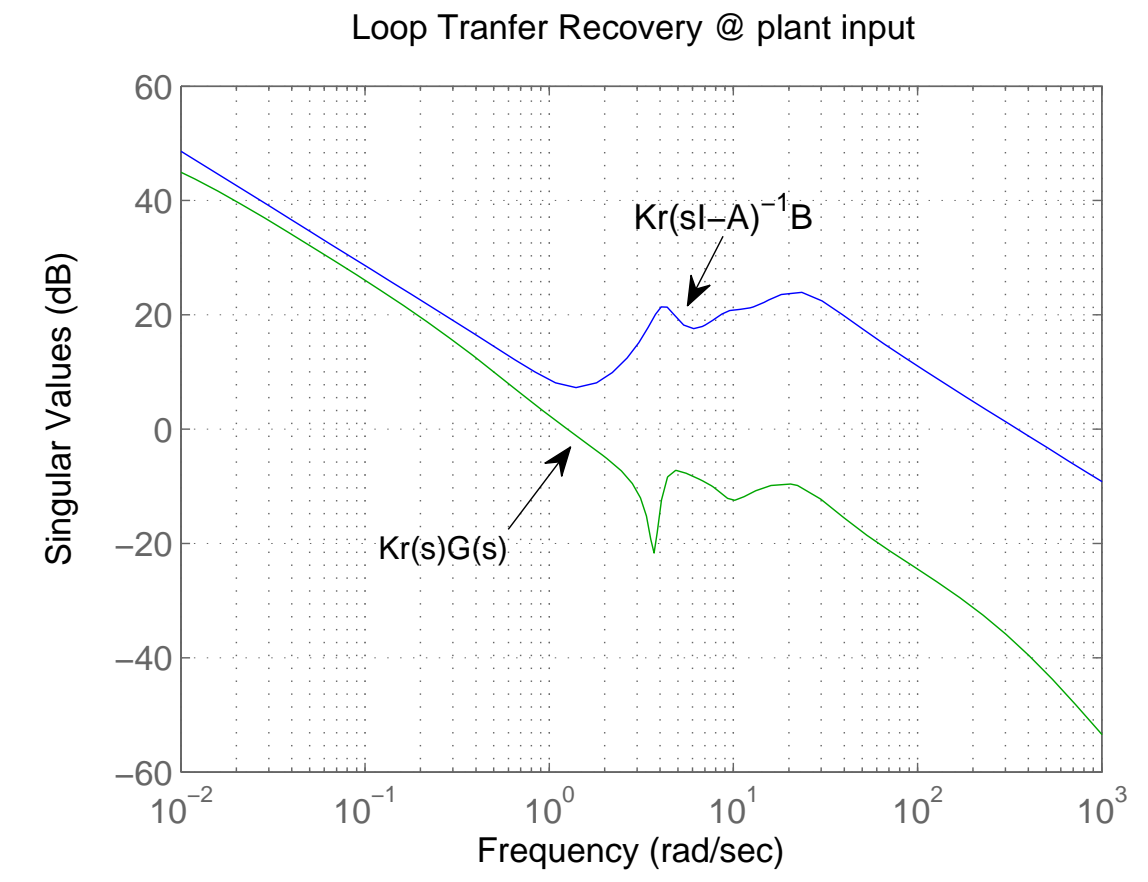

Figure 7

Loop Tranfer Recovery @ plant input

Frequency (rad/sec)

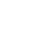




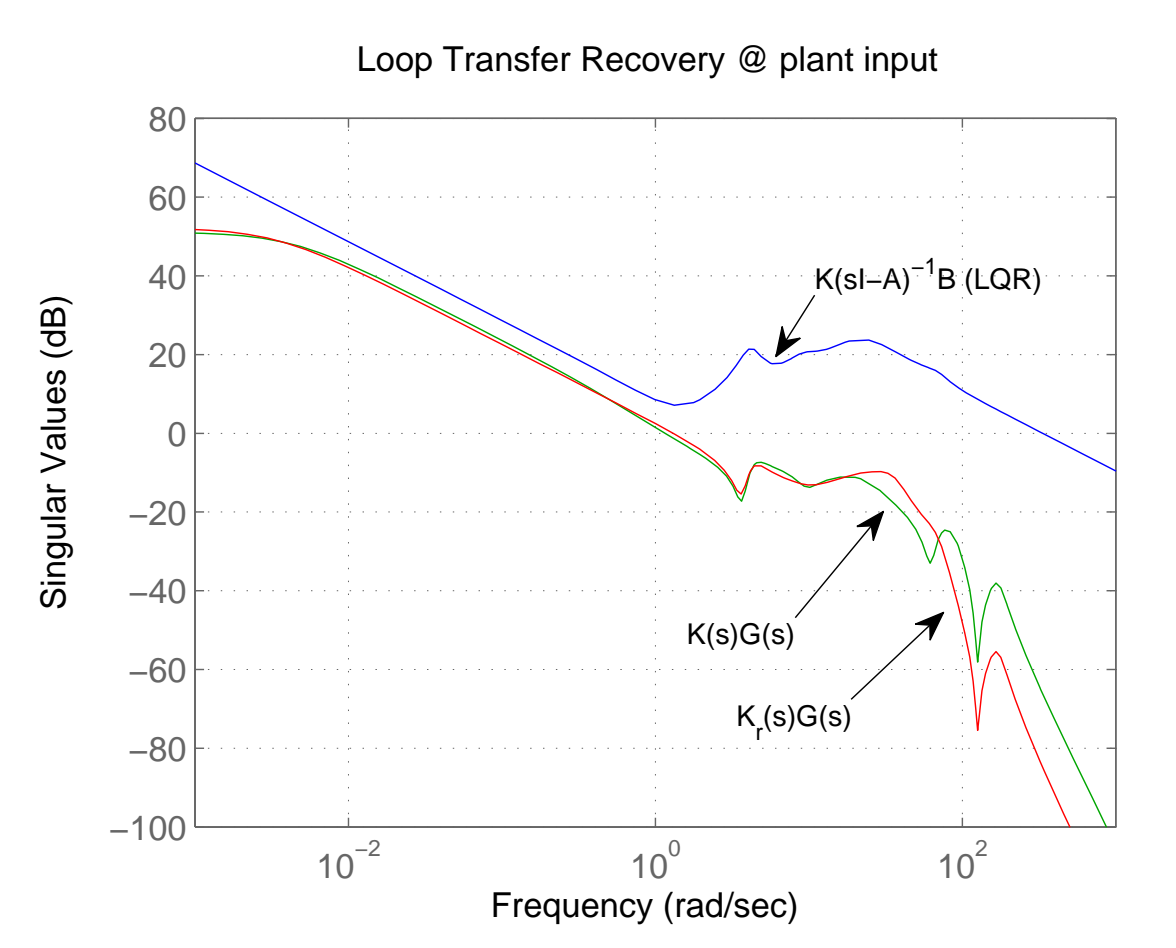

Figure 8

Frequency (rad/sec)

(

$+2$ 


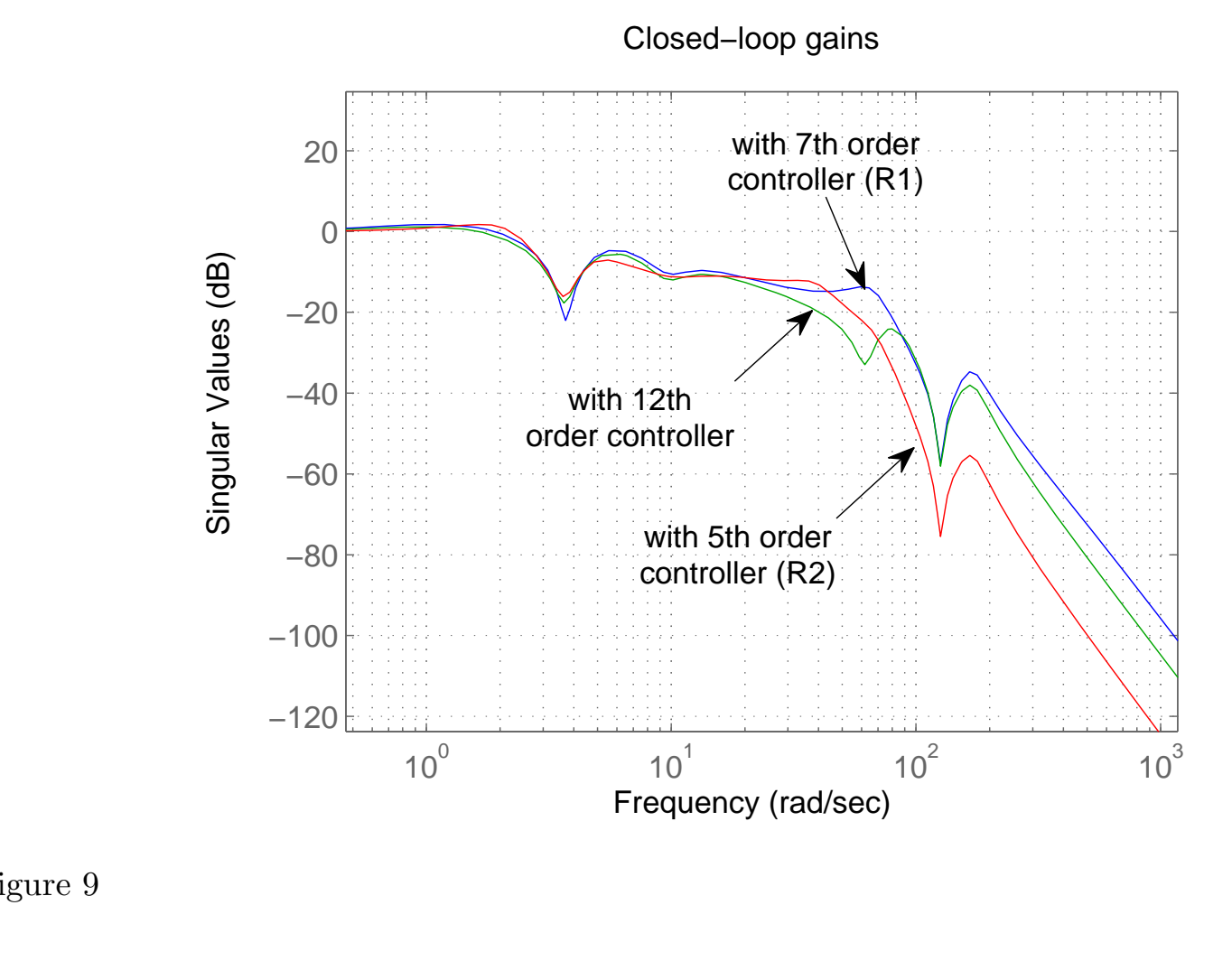

Figure 9

Frequency (rad/sec)

Femers

(20)
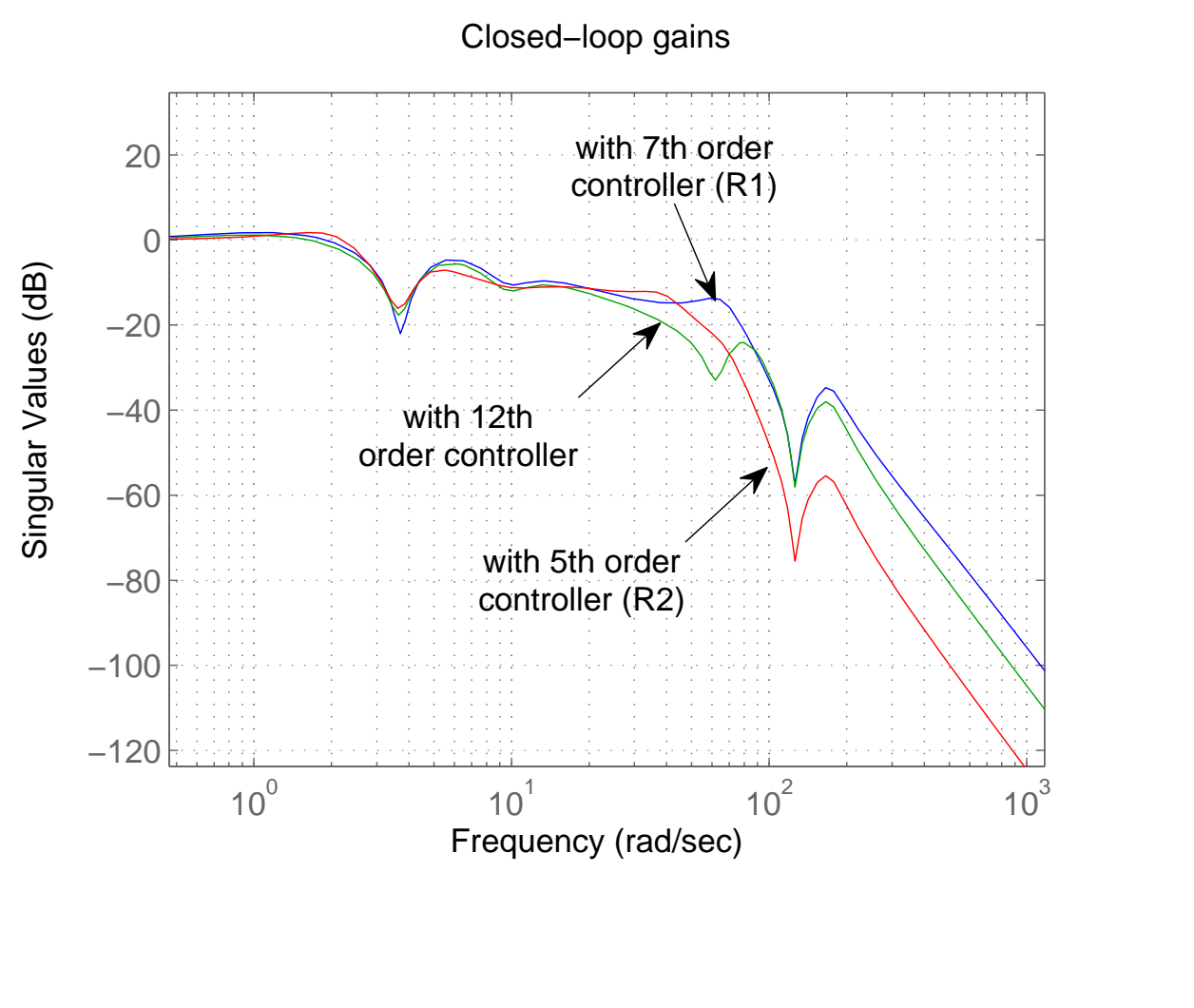


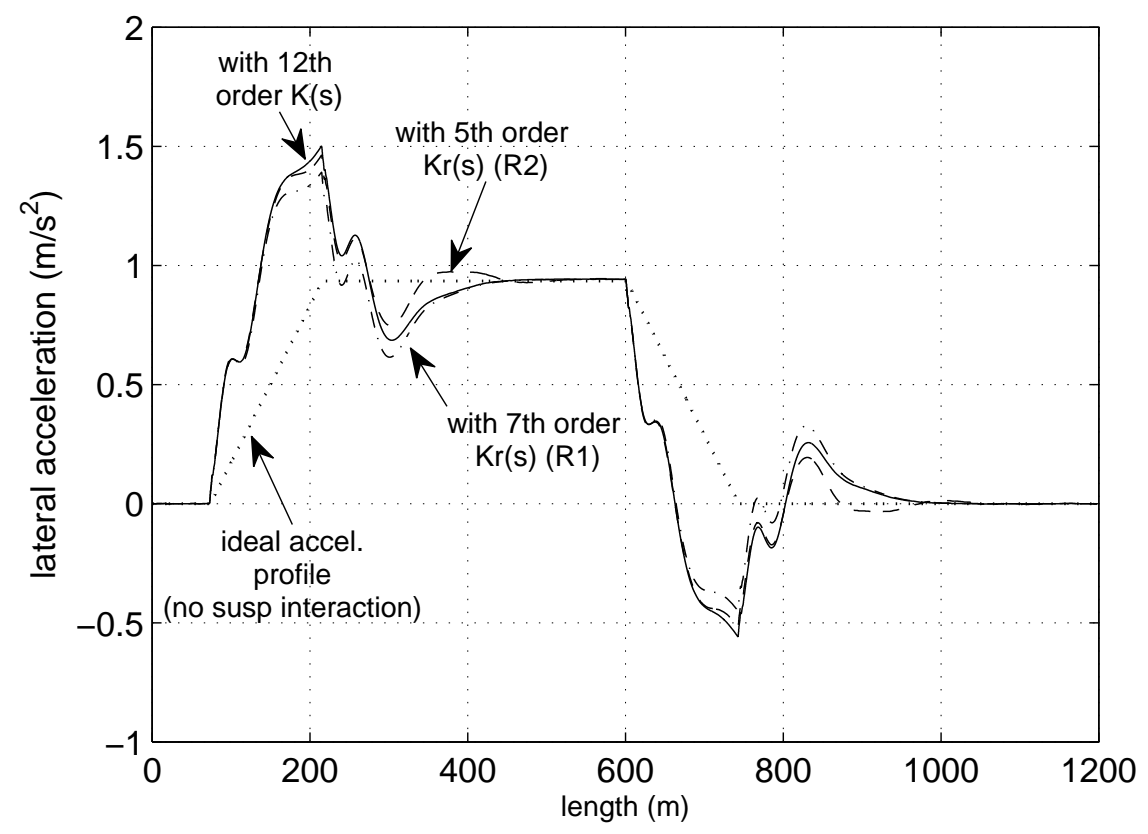

Figure 10a 
